\title{
Tecnologia a serviço da Endodontia: avanços no diagnóstico e tratamento de canais radiculares
}

\section{HUtrevista}

Technology for endodontics: advances in the diagnosis and

treatment of root canals

- Celso Neiva Campos ${ }^{1}$, Alloma de Souza Oliveira Campos $^{2}$ Michelle da Conceição Bellei ${ }^{2}$

${ }^{1}$ Departamento de Clínica Odontológica, Faculdade de Odontologia, Universidade Federal de Juiz de Fora - Juiz de Fora, MG

${ }^{2}$ Programa de Pós-Graduação em Odontologia, Faculdade de Odontologia, Universidade Federal de Juiz de Fora - Juiz de Fora, MG

$\triangle$ Celso Campos

Rua Lindalva de Paula Ribeiro,

240

Bosque Imperial

CEP: 36036-466

Juiz de Fora - MG

○ cncampos@terra.com.br

\section{RESUMO}

A Endodontia é a especialidade da Odontologia que cuida da prevenção, diagnóstico e tratamento das afecções que acometem a polpa dental, a cavidade endodôntica e os tecidos periodontais adjacentes. O tratamento endodôntico, considerado complexo e desgastante para o paciente e operador, durante anos vem sendo foco de atenção para estudiosos da área e indústria especializada. O objetivo deste estudo de revisão de literatura foi apresentar, de forma sucinta, a evolução tecnológica dedicada à Endodontia nas últimas décadas e suas consequências na atualidade. Muitos foram os estudos que acarretaram no desenvolvimento de aparelhos, instrumentos, materiais e técnicas hoje utilizadas visando a simplificação, agilização e aumento da taxa de sucesso do tratamento endodôntico. Entre os principais incrementos na Endodontia podemos citar os modernos instrumentos endodônticos, os sofisticados motores e aparelhos controlados eletronicamente e alguns materiais. Esses, modificaram significativamente a forma de realizar algumas etapas do tratamento como a odontometria, o preparo químico-mecânico, a obturação, o reparo de perfurações e, ainda, o diagnóstico por imagem, tornando a Endodontia mais segura, eficiente, rápida, com maior previsibilidade e menos cansativa para profissional e paciente.

Palavras-chave: endodontia, desenvolvimento tecnológico, tratamento do canal radicular, automação

\section{ABSTRACT}

Endodontics is the specialty of Dentistry concerned with prevention, diagnostics and treatment of affections of the dental pulp, endodontic cavity and the adjacent periodontal tissues. Endodontic treatment, considered complex and exhausting for the patient and operator, has for years been the focus of attention of scholars in this area of expertise and specialized industry. The purpose of this literature review study was to present, succinctly, the technological evolution dedicated to endodontics in the last decades and their consequences at the present time. Many studies have led to the development of devices, instruments, materials and techniques used today to simplify, streamline and increase the success rate of endodontic treatment. Among the main improvements in endodontics, we can mention the modern endodontic instruments, sophisticated motors, electronically-controlled devices and some materials. These significantly modified the way to perform some treatment steps, such as odontometry, chemo-mechanical preparation, filling, perforation repair, and imaging diagnosis, making endodontics safer, more efficient, faster, with greater predictability and less tiring for the professional and patient.

Keywords: endodontics, technological development, root canal therapy, automation 


\section{INTRODUÇÃO}

A Endodontia é a especialidade da Odontologia que cuida da prevenção, diagnóstico e tratamento das afeç̧ões que acometem a polpa dental, a cavidade endodôntica e os tecidos periodontais adjacentes. O tratamento dos canais radiculares, meio utilizado para cuidar dessas afeç̧ões, sempre foi considerado como de grande complexidade e delonga, exigindo do operador muita habilidade, paciência e persistência. Tal característica deve-se principalmente ao fato dos canais radiculares apresentarem, na maioria dos casos, anatomia complexa, com curvaturas, atresias, ramificações e calcificações, associada à impossibilidade de visualização de sua extensão, o que sempre foi feito, com muitas limitações, por meio das radiografias periapicais (SEMAAN, et al., 2009).

Desde o início do século passado até os anos 90, a Endodontia seguia desenvolvendo-se, técnica e cientificamente no sentido de minimizar essas dificuldades, mas com resultados pouco significativos. Considerada como a fase mais trabalhosa do tratamento endodôntico, a instrumentação ou preparo dos canais radiculares sempre esteve em foco entre as pesquisas (HULSMANN; PETERS; DUMMER, 2005). Em 1961, Ingle propôs a padronização dos instrumentos endodônticos, antes fabricados em aço carbono e posteriormente em aço inoxidável, um grande passo para a indústria especializada. Durante esse tempo as limas endodônticas sofreram alterações em suas configurações, seja no design (HASSAN; ROSHDY; ISSA, 2018), na lâmina de corte, formato da ponta, seção transversal da haste e conicidade (KUZEKANANI, 2018).

Incansáveis, sempre foram os esforços na busca de métodos e técnicas que promovessem uma eficiente desinfeç̧ão do sistema de canais radiculares, fator fundamental para o sucesso do tratamento endodôntico, assim como estudos sobre materiais, biocompatibilidade e microbiologia (KISHEN et al., 2016).

Nas últimas três décadas, aproximadamente, as pesquisas e a dedicação no desenvolvimento de novas tecnologias permitiram uma evolução significativa da Endodontia, revolucionando os métodos e técnicas tanto para tratar o canal radicular, quanto para sua visualização e diagnóstico por imagem (TORABINEJAD et al., 1995). Nesse âmago, as limas de liga de níqueltitânio (NiTi) e suas variáveis, altamente flexíveis, os diferentes sistemas motorizados de instrumentação de canais radiculares, os localizadores apicais eletrônicos, a terapia fotodinâmica, a tomografia computadorizada de feixe cônico, cimento MTA, o uso do microscópio e a irrigação ultrassônica.

Destarte, o objetivo deste trabalho foi realizar uma revisão de literatura abordando alguns destes avanços tecnológicos e suas respectivas contribuições e tendências no preparo do sistema de canais radiculares.

\section{REVISÃO DE LITERATURA}

\section{Instrumentação dos canais radiculares}

Até o final da década de 80 , muitos foram esforços e estudos sobre aparelhos, instrumentos e técnicas para instrumentação dos canais radiculares, todavia, não podemos destacar nenhum que represente um marco nesta difícil e importante etapa do tratamento endodôntico. Alguns tiveram destaque e mantêm sua importância até a atualidade como a padronização dos instrumentos (limas) manuais por Ingle (1961). Entretanto, um grande avanço, foi dado pela introdução dos instrumentos de NiTi (WALIA et al., 1988), que conferem até três vezes mais flexibilidade às limas, assim como maior resistência à fratura em relação às de aço inoxidável, que eram utilizadas na época e, por sua vez, substituíram os instrumentos em aço carbono (HULSMANN; PETERS; DUMMER, 2005).

Desde então, houve uma evolução na mecanização do preparo do sistema de canais radiculares (SCR) com desenvolvimento rápido de várias gerações de instrumentos e equipamentos pela indústria como a inserção do movimento rotatório em limas de NiTi logo em seguida, em 1994, por Johnson e McSpadden, (HAAPASALO; SHEN, 2013). Destes sistemas de instrumentos, fazem parte o ProTaper ${ }^{\circledR}$ (Dentisply/ Maillefer), sistema K3 $₫$ (Sybron Keer), Quantec ${ }^{\circledR}$, EndoSequence $₫$ e BioRace ${ }^{\circledR}$ (KUZEKANANI, 2018).

Em 2007, a liga de NiTi, por meio de um tratamento térmico, evoluiu para a liga denominada M-wire, que resultou em melhorias na resistência à fadiga dos instrumentos proporcionando mais segurança em relação à fratura durante o preparo mecânico (KUZEKANANI, 2018). Dentre eles pode-se destacar o Profile Vortex. Em 2008, Ghassan Yared iniciou um trabalho preliminar utilizando o instrumento ProTaper F2 em movimento reciprocante, na tentativa de otimizar um único instrumento a modelar qualquer tipo de canal (YARED, 2008). Porém, era necessária uma mudança na forma do instrumento para esse tipo de movimentação, definido como um movimento repetitivo de ida e volta, nos sentidos horário e anti-horário (HAAPASALO; SHEN, 2013). Em 2010, foi a vez da liga CM-Wire, que tinha como característica maior flexibilidade e memória de forma, como o HyFlex CM e o K3FX. Assim, em 2011, foram inseridas no mercado as limas com conceito de uso único, WaveOne (Dentisply) e Reciproc (VDW), ambas de liga M-wire e movimento reciprocante. Com o avanço da instrumentação mecanizada, houve a necessidade da criação do chamado Glide Path, definido como um préalargamento do canal radicular antes da instrumentação mecanizada para manter o formato original do canal e diminuir erros de instrumentação, como transporte apical e descentralização apical (HULSMANN; PETERS; DUMMER, 2005). 
A partir de então surgiram inúmeros instrumentos com movimentação rotatória e reciprocante e até mesmo instrumentos com formato e tipo de movimento diferentes, como exemplos, o Self Adjusting File (SAF) (HAAPASALO; SHEN, 2013), o OneShape, instrumento único em movimento rotatório, Revo-S e XP-endo Shaper (FKG Dentaire), recém lançada no mercado com movimento contínuo e mudança de fase de acordo com a temperatura (HASSAN; ROSHDY; ISSA, 2018; KUZEKANANI, 2018).

Para realizar o preparo químico-mecânico do canal radicular é de suma importância estabelecer um comprimento de trabalho (CT), que sempre foi realizado por meio de radiografias periapicais. Este método acarretava em muitas dúvidas quanto ao verdadeiro ponto onde devia-se estabelecer o limite apical da instrumentação. Durante anos, a comunidade científica discutiu sobre esse limite, sempre gerando muitas discussões. (GORDON; CHANDLER, 2004).

O primeiro localizador apical eletrônico foi desenvolvido por Sunada (1962), seguido pela evolução de quatro gerações de equipamentos até os dias atuais (OTHEEM et al., 2018). Os representantes mais conhecidos da primeira geração foram Exact-A-Pex ${ }^{\circ}$, Endometer $\AA$, Neosono $D \AA$, Neosono $M \circledR$, Foramatron $\AA$ (O'NEILL, 1974); da segunda geração foi o Endocater $($; da quarta geração, o Root ZX (J. Morita, Kyoto, Japan). Os primeiros aparelhos apresentavam limitações quanto à precisão das mensurações devido à presença de fluídos, de tecido pulpar e da necessidade de isolamento do instrumento endodôntico durante a medição (GORDON; CHANDLER, 2004). Também se tornaram conhecidos o Root ZX mini (J. Morita Corporation), de quinta geração, e o Apex ID (SybronEndo) e CanalPro (ColteneEndo), de sexta geração. O uso do localizador eletrônico evita consequências desagradáveis como formação de degraus nas paredes dos canais, assim como instrumentação e obturação inadequadas, perfuração radicular e sintomatologia pós-operatória (FERREIRA; FRONER; BERNARDINELI, 1998). Além disso, o uso da radiografia é limitado, já que a precisão da constrição apical é difícil de ser encontrada pela bidimensionalidade das radiografias periapicais (FERREIRA; FRONER; BERNARDINELI, 1998; GORDON; CHANDLER， 2004; OTHEEM et al., 2018).

\section{Desinfecção dos canais radiculares}

A desinfecção dos canais radiculares consiste na etapa que complementa a parte mecânica do preparo e modelagem do sistema de canais radiculares (SCR), visto que permite a limpeza também das áreas não tocadas pelos instrumentos (CESARIO et al., 2018). Porém, esta etapa pode apresentar algumas dificuldades que podem ser minimizadas através do meio utilizado para a desinfecção. Uma dessas dificuldades é a formação de smear layer, que impede o contato do irrigante nas paredes do canal (CIUCCHI; KHETTABI; HOLZ, 1989) e sua ação para o interior dos túbulos dentinários; a permanência de micro-organismos, seja por complexidade anatômica ou pela irrigação inadequada; e o vapor lock, que corresponde ao ar preso na porção apical do SCR (GU et al., 2009). O uso alternado do hipoclorito de sódio $(\mathrm{NaOCl})$ e o ácido etilenodiaminotetracético (EDTA) tem sido recomendado pela eficiência de sua ação nas paredes do canal como desproteinizante e na remoção de smear layer (CIUCCHI; KHETTABI; HOLZ, 1989).

A irrigação convencional, realizada manualmente através de agulhas/seringas/cânulas, mesmo sendo amplamente aceita e utilizada no dia a dia clínico pela relação custo/benefício e pela segurança ao apresentar menos risco de extravasamento de irrigante aos tecidos periapicais, mostra falhas pelo fluxo do irrigante não se estender muito além da ponta da agulha, o que fomentou pesquisas e tecnologias para melhorar a qualidade e efetividade do preparo químico do SCR (GU et al., 2009).

Durante a década de 1990, foram inseridos no mercado métodos alternativos para o desbridamento do SCR, onde os dividimos em dois modos de ação: técnica de agitação de irrigantes manual e técnica de agitação de irrigantes de forma mecanizada. Dentre as técnicas manuais temos a irrigação convencional, com agulhas/cânulas; as escovas para canal radicular (GU et al., 2009), como a Endobrush (C\&S Microinstruments Ltd, Markham, Ontario, Canada) e Navitip FX (Ultradent Products Inc, South Jordan, UT); e a agitação mecânica manual, realizada pela ativação através de guta-percha ajustada ao forame apical. Dentre a técnica de agitação de irrigante de forma mecanizada, temos as escovas rotatórias, como o Ruddlebrush e o Canalbrush (Coltene Whaledent); a irrigação através do movimento rotatório, como o Quantec-E (SybronEndo), XP-endo finisher (FKG Dentaire) e Easy Clean (Easy Dental Equipment); pelo Sonic, com EndoActivator e o Rispisonic (Medidenta International); pelo Ultrasonic, com movimento contínuo (UI - ultrasonic irrigation) ou intermitente (PUI - Passive Ultrasonic Irrigation); e pelo dispositivo de alternância de pressão, com o EndoVac e o RinsEndo (Durr Dental).

A irrigação passiva ultrassônica (PUI) obteve destaque, já que grande parte dos estudos apontaram para uma eficácia superior àquela apresentada pela irrigação passiva convencional por seringa/agulha na remoção de restos de tecido pulpar e smear layer (SABINS; JOHNSON; HELLSTEIN, 2003; CESARIO et al., 2018). Nesta técnica, o irrigante é introduzido no SCR pela irrigação passiva seringa/agulha e ativado com o uso de um instrumento oscilante ultrassônico. A eficácia desta técnica se deve à agitação do irrigante e seu volume serem muito maiores do que na técnica convencional (LEE; WU; WESSELINK, 2004).

Além destes, merece destaque na desinfecção dos canais radiculares a terapia fotodinâmica (PDT 
- Photodynamic Therapy) que é baseada no agente fotossensibilizante não tóxico, conhecido como fotossensibilizador, que quando ativado por uma luz, gera radicais livres citotóxicos às células do tecido alvo (DOUGHERTY et al., 1998). A PDT é um novo método que objetiva aumentar a eficácia da desinfecção de canais radiculares, através da irradiação com laser de baixa intensidade, por uma fibra ótica introduzida nos canais radiculares previamente corados pelo fotossensibilizador, geralmente o azul de metileno. Isso leva à ruptura das paredes celulares bacterianas e à destruição dos microorganismos (LACERDA et al., 2016). O primeiro laser designado especificamente à odontologia foi introduzido em 1989 e uma variedade de lasers são descritos, como o laser de dióxido de carbono, Laser Nd:YAG; Er:YAG e Er:YSGG, Argon, Krypton e o laser diodo, sendo o último o mais utilizado na Endodontia (MARKOVIĆ et al., 2015). Alguns estudos concluíram que a PDT pode ser um adjunto ao tratamento químico-mecânico convencional, pela redução microbiana no interior dos canais radiculares, principalmente de bactérias gram-positivas, que são os microbiotas mais resistentes e presentes no insucesso da terapia endodôntica (LACERDA et al., 2016; MARKOVIĆ et al., 2015).

\section{Diagnóstico por imagem}

Várias modalidades de captura de imagens são utilizadas na Endodontia clínica atual, como a radiografia convencional, a radiografia digital e a tomografia computadorizada de feixe cônico (TCFC). A radiografia convencional possui como características a bidimensionalidade, ser dependente do fator de exposição, processamento químico e filme intraoral (KISHEN et al., 2016). As radiografias digitais surgiram na década de 80 , trazendo melhorias em relação ao sistema anterior, como diminuição da exposição do paciente à radiação, capacidade de interpretação imediata da imagem, facilidade para arquivar e compartilhar as imagens, manipulação das imagens para melhoria das propriedades como contraste e brilho no programa computacional, e eliminação da etapa de processamento químico (KISHEN et al., 2016). Os sistemas de captura digital incluem sensores eletrônicos digitais e são criados por um dispositivo de carga acoplada (CCD) ou um semicondutor complementar de óxido de metal (CMOS), enquanto os sistemas indiretos usam placa de fósforo fotoestimulável (PSP) (KISHEN et al., 2016).

A tomografia computadorizada de feixe cônico (TCFC) foi introduzida na área odontológica através do estudo preliminar de Mozzo et al. (1998) chamado NewTom-9000, onde relataram alta acurácia das imagens, assim como baixa dosagem em relação à tomografia computadorizada convencional. Além disso, proporciona uma imagem tridimensional, com pouca distorção e facilita a interpretação, diagnóstico e prognóstico (BAHCALL, 2015; KISHEN et al., 2016). Agregou uma série de benefícios à Endodontia como visualização de fraturas radiculares (DE MARTIN E SILVA et al., 2018), reabsorções interna e externa, extensão de lesões periapicais, proximidade com seio maxilar, desvios e perfurações. A TCFC não é uma substituta das radiografias convencionais e digitais, mas sim um exame complementar quando houver limitação para as radiografias periapicais (VERNER et al., 2017; BAHCALL, 2015). Todavia, ela também apresenta limitações, como dificuldade de visualização das imagens na presença de artefatos metálicos (DE MARTIN E SILVA et al., 2018) e menor resolução de imagem em comparação às radiografias periapicais (KISHEN et al., 2016).

O microscópio eletrônico é um equipamento complementar de diagnóstico clínico por meio da magnificação de imagem da área tratada, que permite a identificação das estruturas com maior precisão e a realização de tratamentos com maior previsibilidade (TANG; LI; YIN; 2010; PECORA; PECORA; 2015). Ele também auxilia na localização de canais radiculares calcificados, na visualização de linhas finas de fraturas verticais ou trincas, na detecção de microinfiltrações, além de permitir cavidades de acesso com menor amplitude (TANG; LI; YIN; 2010).

\section{Material reparador}

Durante várias décadas, muitos materais foram desenvolvidos para a Endodontia, mas cabe destaque especial ao agregado trióxido mineral, mais conhecido como MTA (Mineral Trioxide Agregate). Ele foi desenvolvido na Universidade Loma Linda na década de noventa e patenteado por Torabinejad et al (1995). Logo recebeu a aceitação pela Federal Drug Administration (FDA) dos EUA e tornou-se comercialmente disponível como ProRoot MTA (Tulsa Dental Products, Tulsa, OK, EUA). Até recentemente, duas formas comerciais de MTA estavam disponíveis (ProRoot MTA) nas formas cinza ou branca (CAMILLERI; FORD, 2006).

O MTA é derivado de um composto original do cimento Portland. Embora esse composto seja similar em alguns aspectos, o cimento Portland e o cimento odontológico não são idênticos. O MTA passa por processamento e purificação adicionais. Os produtos MTA, quando comparados aos cimentos Portland, têm um tamanho médio de partícula menor e contêm menos metais pesados tóxicos (ISLAM, CHNG e YAP, 2006).

Sua criação foi um passo importante para a Endodontia, visto que muitos problemas de origem patológica ou iatrogênica, como por exemplo, as perfurações radiculares, passaram a ser possivelmente solucionáveis com o advento deste cimento endodôntico (TORABINEJAD et al., 1995).

Alguns estudos (TORABINEJAD et al., 1995; TANG; LI; YIN; 2010) mostraram as características positivas do MTA. Várias propriedades são de grande valia para o resultado desejável no uso desse cimento. Destacam-se a biocompatibilidade, bioatividade, hidrofilicidade (uso na 
presença de umidade), radiopacidade e baixa solubilidade. A alta biocompatibilidade estimula respostas ótimas de cura. Isso tem sido observado histologicamente com a formação de novo cemento na área dos tecidos perirradiculares, ou seja, ocorre a indução de reparo tecidual mineralizado e uma baixa resposta inflamatória com formação de ponte no espaço pulpar (TORABINEJAD et al., 1995).

Atualmente, o MTA tem sua indicação voltada principalmente para as reabsorções perfurantes, fechamentos apicais, retro-obturações nas cirurgias parendodônticas, apecificações, assim como nos recentes tratamentos por Endodontia regenerativa (TANG; LI; YIN; 2010).

\section{Obturação dos canais radiculares}

O objetivo da obturação dos canais radiculares é promover um ambiente que impede a proliferação e crescimento de bactérias residuais e inibir o aparecimento de novas bactérias (CATHRO; LOVE, 2003). A técnica de cone único e a técnica da condensação lateral e vertical são técnicas pioneiras na Endodontia e utilizadas até os dias de hoje (SCHILDER, 1967). Mas, devido às suas limitações, passaram por modificações com objetivo de cumprir as necessidades de um bom resultado para o preenchimento correto do SCR.

O compactador de McSpadden foi uma destas modificações introduzido em 1978 (MCSPADDEN, 1980), que corresponde a um instrumento de aço inoxidável que utiliza energia friccional através do movimento rotatório para aquecer a guta-percha e compactá-la apicalmente. Porém, é um instrumento propenso à fratura e pode extravasar material obturador com facilidade através do ápice (CATHRO; LOVE, 2003). A técnica híbrida de Tagger e a técnica híbrida de Tagger modificada, utilizando condensadores manuais (TAGGER, 1984), também vieram para aprimorar o conceito de Schilder (1967), onde acreditou-se que o preenchimento tridimensional por todo espaço pulpar, incluindo ramificações, deveria ser atingido.

Buchanan, em 1996, apresentou o System B (Analytic Sybron Dental Specialties, Orange, CS, USA), um aparelho que funciona como um condensador de onda contínua com fonte de calor, com variedade em tamanho de pontas. Outros sistemas obturadores que merecem destaque são o sistema Obtura II, formado por um componente que injeta guta-percha termoplastificada no interior dos canais radiculares a uma temperatura de 150 a $200^{\circ} \mathrm{C}$, e o sistema Thermafill, que consiste em um flexível suporte uniformemente revestido por guta-percha aquecida que penetra no interior dos canais radiculares (HARGREAVES; LOUIS, 2011). Ele apresenta como vantagens o curto período para obturação e uma melhor adaptação da gutapercha aquecida à anatomia interna dos canais radiculares (BHANDI; SUBHASH, 2013). Porém, a sobrecarga do canal radicular devido ao aumento do fluxo do material termoplastificado e a contração do material após seu esfriamento foram relatados como desvantagens desse sistema (CATHRO; LOVE, 2003).

Os sistemas e aparelhos desenvolvidos para obturação dos canais radiculares se mostraram complexos quanto ao manuseio, além do custo elevado do material de reposição. Exceção para o compactador de McSpadden, por se tratar de um instrumento de baixo custo, fácil manuseio e rápida termoplastificação mecânica.

\section{DISCUSSÃO}

Atualmente, a humanidade está cercada de tecnologias inovadoras, das mais diferentes formas, visando facilitar nossas tarefas diárias com mais conforto, agilidade, segurança e, às vezes, com menor custo operacional. A Endodontia, assim como a Odontologia, estão inseridas nesse contexto, por meio de uma série de avanços conquistados, principalmente, nas duas últimas décadas.

O grande marco deste salto tecnológico pode ser descrito como a introdução dos sistemas motorizados para instrumentação dos canais radiculares, conjugado ao desenvolvimento de novas ligas metálicas para confecção das limas endodônticas, como as de NiTi e M-Wire (WALIA, 1988; KUZEKANANI, 2018). Dessa associação, surgiu um novo conceito em preparo de canais radiculares, com movimentos automatizados rotatório ou reciprocante, que possibilita uma instrumentação de qualidade com mais segurança e rapidez, principalmente nos casos de canais com curvaturas significativas (HAAPASALO; SHEN, 2013). Todavia, com a redução do tempo de trabalho, impedese que a substância irrigante permaneça por mais tempo em contato com a superfície radicular. Com objetivo de contornar essa deficiência algumas alternativas foram desenvolvidas e têm sido propostas como a ativação da substância irrigante por vibração sônica ou ultrassônica. Também a terapia fotodinâmica, por meio dos aparelhos de laser de baixa potência, tem sido proposta como adjuvante (LACERDA et al., 2016).

Para um tratamento endodôntico seguro, é de suma importância o respeito ao ligamento periodontal e, para isso, torna-se necessária uma precisão do comprimento de trabalho a ser estabelecido durante o tratamento, o que até a década passada era obtido apenas por meio de radiografias periapicais, que devido a sobreposição de estruturas e distorções é impreciso (KISHEN et al., 2016). Com o advento dos localizadores apicais eletrônicos este procedimento tornou-se rápido, simples e muito mais seguro, devido à sua maior acurácia na determinação do limite apical de instrumentação (OTHEEM et al., 2018), assim como na constatação de canais e perfurações radiculares.

Outro recurso de excelência para a Endodontia foi a TCFC, cada vez mais utilizada, possibilita uma maior segurança no diagnóstico e planejamento de tratamentos clínicos e cirúrgicos (KISHEN et al., 2016). Este recurso, praticamente eliminou a cirurgia parendodôntica exploratória, um procedimento muito comum em casos duvidosos sobre a 
origem de problemas endodônticos. Como ferramenta de magnificação de imagem, o microscópio clínico já é utilizado há pelo menos duas décadas. Apesar de sua grande utilidade na visualização da cavidade pulpar, terço cervical do canal e nas cirurgias parendodônticas (TANG; LI; YIN; 2010; PECORA; PECORA; 2015), ainda possui limitações como o alto custo, versatilidade e impossibilidade de visualização após curvaturas.

Apesar de não ser propriamente um avanço tecnológico, não se pode deixar de citar o MTA, material reparador que mudou o paradigma da Endodontia no que se refere a perfurações, reabsorções radiculares e terapia regenerativa (TANG; LI; YIN; 2010). Todavia, as tecnologias hoje empregadas nas pesquisas que 0 envolvem têm sido responsáveis pela sua aplicação cada vez mais ampla.

Muitos sistemas e equipamentos como o Obtura II, Thermafill, System B foram inseridos no mercado como novas tecnologias para a obturação dos canais radiculares (BHANDI; SUBHASH, 2013), mas devido ao alto custo, complexidade de manuseio, dificuldade de obtenção de material de reposição, não obtiveram boa adesão junto aos profissionais brasileiros. Para a termoplastificação mecânica da guta-percha hoje tem-se utilizado mais comumente o Compactador de McSpadden e as técnicas de Schilder e Híbrida de Tagger Modificada.

Por fim, pode-se afirmar que foram muitas as conquistastecnológicas para Endodontia queagregaram segurança, qualidade, conforto, rapidez, entre tantas outras vantagens para o tratamento endodôntico. Entretanto, cabe a cada um, como profissional da área, e em cada caso específico, considerando riscos, benefícios, vantagens e desvantagens, estabelecer 0 momento de sua utilização.

\section{CONCLUSÃO}

Nos últimos anos houve um significativo avanço tecnológico com consequente transformação na Endodontia, tanto no desenvolvimento de novas técnicas, com a introdução de aparelhos quanto de novos materiais. Tais avanços reduziram drasticamente o tempo operatório do tratamento, gerando maior conforto para o paciente e menor estresse para o profissional. Ressalta-se que a tecnologia deve ser utilizada com bom senso e demanda conhecimento e treinamento do profissional antes de utilizá-las.

\section{REFERÊNCIAS}

BAHCALL, J. Today's endodontic therapy driven by advances in technology, changes in thinking. Compendium, v. 36, n. 5, p. 378-379, may. 2015.
BHANDI, S. H.; SUBHASH, T. S. Comparative evaluation of sealing ability of three newer root canal obturating materials guttaflow, resilon and thermafil: an in vitro study. Journal of International Oral Health, v. 5, n. 1, p. 54-65, feb. 2013.

BUCHANAN, L. S. The continuous wave of obturation. Dentistry Today, v. 15 , n. 1, p. 64-7, jan. 1996.

CAMILLERI, J.; FORD, T.R.P. Mineral trioxide aggregate: a review of the constituents and biological properties of the material. International Endodontic Journal, v.39, n. 10, p.747-754, oct. 2006.

CATHRO, P. R.; LOVE, R. M.; Comparison of microseal and system b/ obtura II obturation techniques. International Endodontic Journal, v. 36, n. 2, p. 876-882, dec. 2003.

CESARIO, F. et al. Comparisons by microcomputed tomography of the efficiency of diferente irrigation techniques for removing dentinal debris from artificial groove. Journal of Conservative Dentistry, v. 21, n. 4, p. 383-387, jul./aug. 2018.

CIUCCHI, B.; KHETTABI, M.; HOLZ, J. The effectiveness of different endodontic irrigation procedures on the removal of the smear layer: a scanning electron microscopic study. International Endodontic Journal, v. 22, n. 1, p. 21-28, jan. 1989.

DE MARTIN E SILVA, D. et al. Diagnosis of mesiodistal vertical root fractures in teeth with metal posts: influence of applying filters in cone-beam computed tomography images at different resolutions. Journal of Endodontics, v. 44, n. 3, p. 470-474, mar. 2018.

DOUGHERTY, T. J. et al. Photodynamic therapy. Journal of the National Cancer Institute, v. 90, n. 12, p. 889-905, jun. 1998.

FERREIRA, C.; FRONER, I.; BERNARDINELI, N. Utilização de duas técnicas alternativas para localização do forame apical em Endodontia: avaliação clínica e radiográfica. Revista de Odontologia da Universidade de São Paulo, v. 12, n. 3, p. 241246, jul. 1998.

GORDON, M.P.; CHANDLER, N.P. Electronic apex locators. International Endodontic Journal, v. 3, n. 7, p. 425-437, jul. 2004.

GU, L.; et al. Review of contemporary irrigant agitation techniques and devices. Journal of Endodontics, v. 35, n. 6, p. 791-804, jun. 2009.

HASSAN, R.; ROSHDY, N.; ISSA, N. Comparison of canal transportation and centering ability of $x p$ shaper, waveone and oneshape: a cone beam computed tomography study of curved root canals. Acta Odontologica Latinoamericana, v. 31, n. 1, p. 67-74, jun. 2018. 
HAAPASALO, M.; SHEN, Y. Evolution of nickel-titanium instruments: from past to future. Endodontic Topics, v. 29, p. 3-17, nov. 2013.

HARGREAVES, K.; LOUIS, B. Obturation of the cleaned and shaped root canal system .In: COHEN S; BURNS R. C. Pathways of Pulp. 10. ed. St. Louis, MO, USA: Mosby, 2011. p. 349-389.

HULSMANN, M.; PETERS, O. A.; DUMMER, P. M. H. Mechanical preparation of root canals: shaping goals, techniques and means. Endodontic Topics, v. 10, n. 1, p. 30-76, mar. 2005.

INGLE, J. I. A standardized endodontic technique using newly designed instruments and filling materials. Oral Surgery, Oral Medicine, Oral Pathology, v. 14, n. 1, p. 83-91, jan. 1961.

ISLAM, I.; CHNG, H. K.; YAP, A. U. Comparison of the physical and mechanical properties of MTA and portland cement. Journal of Endododontics, v. 32, n. 3, p. 193-197, mar. 2006.

KISHEN, A. et al. Advances in endodontics: potential applications in clinical practice. Journal of Conservative Dentistry, v. 19, n. 3, p. 199-206, jun. 2016.

KQIKU, L.; STADTLER, P. Radiographic versus electronic root canal working length determination. Indian Journal of Dental Research, v. 22, n. 6, p. 777-780, nov./dec. 2011.

KUZEKANANI, M. Nickel-titanium rotary instruments: development of the single-file systems. Journal of International Society of Preventive and Community Dentistry, v. 8, n. 5, p. 386-390, jun./aug. 2018.

LACERDA, M. L. S. et al. Evaluation of the dentin changes in teeth subjected to endodontic treatment and photodynamic therapy. Revista de Odontologia da UNESP. v. 45, n. 6, p. 339-343, nov./dec. 2016.

LEE, S. J.; WU, M. K.; WESSELINK, P. R. The effectiveness of syringe irrigation and ultrasonics to remove debris from simulated irregularities within prepared root canal walls. International Endodontic Journal, v. 37, n. 10, p. 672-678, oct. 2004.

MARKOVIĆ, D.; RAKAŠEVIĆ, D.; TRIŠIĆ, D. Application of highpower diode laser and photodynamic therapy in endodontic treatment - review of the literature. Balkan Journal of Dental Medicine, v. 19, n. 2, p. 71-74, jul. 2015.

MCSPADDEN, J. Self study course for the thermatic condensation of gutta-percha. Toledo, OH, USA: Ranson and Randolph, 1980.

MOZZO, P. A new volumetric CT machine for dental imaging based on the cone-beam technique: preliminary results. European Radiology, v. 8, p. 1558-1564, jun. 1998.
O'NEILL, L.J. A clinical evaluation of electronic root canal measurement. Oral Surgery, Oral Medicine, Oral Pathology, Oral Radiology and Endodontology, v. 38, n. 3, p. 469-473, sep. 1974.

OTHEEM, H. A. et al. Comparative analysis of two electronic apex locators in working length determination using stainless steel and nickel titanium hand files in permanent teeth with simulated apical root resorption - An in vitro study. International Archives of Integrated Medicine, V. 5, N. 5, p.45-51, apr. 2018.

PECORA, G.E.; PECORA, C. N. A new dimension in endo surgery: micro endo surgery. Journal of Conservative Dentistry, v. 18, n. 1, p. 7-14, jan./feb. 2015.

SABINS, R. A.; JOHNSON, J. D.; HELLSTEIN, J. W. A comparison of the cleaning efficacy of short-term sonic and ultrasonic passive irrigation after hand instrumentation in molar root canals. Journal of Endodontics, v. 29, n. 10, p. 674-678, oct. 2003.

SCHILDER, H. Filling root canals in three dimensions. Dental Clinics of North America, v. 11, p. 723-744, 967.

SEMAAN, F. S. et al. Endodontia mecanizada: a evolução dos sistemas rotatórios contínuos. Revista Sul-Brasileira de Odontologia, v. 6, n. 3, p. 297-309, set. 2009.

SUNADA, I. New method for measuring the length of the root canal. Journal of Dental Research, v. 41, n. 2, p. 375-387, mar. 1962.

TAGGER, M. et al. Evaluation of the apical seal produced by a hybrid root canal filling method, combining lateral condensation and thermatic compaction. Journal of Endodontics, v. 10, n. 7, p. 299-303, jul. 1984.

TANG, Y.; LI, X.; YIN, S. Outcomes of MTA as root-end filling in endodontic surgery: a systematic review. Quintessence International, v. 41, n. 7, p. 557-566, jul./aug. 2010.

TORABINEJAD, M. et al. Physical and chemical properties of a new root-end filling material. Journal of Endodontics, v. 21, n. 7, p. 349-353, jul. 1995.

VERNER, F. S. et al. Influence of cone-beam computed tomography filters on diagnosis of simulated endodontic complications. International Endodontic Journal, v. 50, n. 11, p. 1089-1096, nov. 2017.

WALIA, H.; BRANTLEY, W. A.; GERSTEIN, H. An initial investigation of the bending and torsional properties of nitinol root canal files. Journal o Endodontics, v. 14, n. 7, p. 346-351, jul. 1988.

YARED G. Canal preparation using only one Ni-Ti rotary instrument: preliminary observations. International Endodontic Journal, v.41, n. 4, p. 339-344, apr. 2008. 